\title{
Adsorción por lote y en una columna de lecho fijo del colorante B39 sobre carbón activado granular
}

\author{
Batch adsorption and fixed bed column of the B39 dye on granular \\ activated carbon B39
}

\author{
Grey C. Castellar Ortega ${ }^{1}$, Beatriz M. Cardozo Arrieta ${ }^{2}$, Jhon F. Suarez Guerrero ${ }^{3}$, Javier E. Vega Taboada ${ }^{3}$ \\ ${ }^{1} \mathrm{Mg}$. Ciencias Química. Docente Investigador. Dirección de Investigaciones y Transferencias. Universidad Autónoma del Caribe. \\ E-mail: greycastellar@gmail.com \\ ${ }^{2}$ Esp. Ciencias Ambientales. Docente Tiempo Completo. Universidad Autónoma del Caribe. \\ ${ }^{3}$ Ingeniero Mecánico. Facultad de Ingeniería. Universidad Autónoma del Caribe.
}

Recibido 22/05/13, Aceptado 22/06/2013

\section{RESUMEN}

Los colorantes están usualmente presentes en los efluentes acuosos de muchas industrias, como la textil, del cuero, el papel, la imprenta y cosméticos. La efectividad de la adsorción de colorantes presentes en aguas residuales, empleando adsorbentes de bajo costo, ha permitido la extensión de esta técnica de remoción, por encima de otros métodos de tratamiento. Este trabajo investigó la capacidad de adsorción de un carbón activado comercial (CA) en la remoción del colorante reactivo Blue 39 (B39) en disolución acuosa a $25^{\circ} \mathrm{C}$. Los datos experimentales del estudio en lote realizado a diferentes $\mathrm{pH}(6-8)$ y concentraciones iniciales de B39 (5-200 $\left.\mathrm{mg} \mathrm{dm}^{-3}\right)$, se ajustan satisfactoriamente al modelo de isoterma de Freundlich, presentando una capacidad máxima de adsorción en monocapa de $17,7 \mathrm{mg} \mathrm{g}^{-1}$ a pH 8 . El estudio dinámico a diferentes alturas del lecho (1, 3 y $\left.5 \mathrm{~cm}\right)$, flujos volumétricos $\left(1\right.$ y $5 \mathrm{~cm}^{3}$ $\mathrm{min}^{-1}$ ) y concentración inicial de $5,5 \mathrm{mg} \mathrm{dm}^{-3}$, muestra que el tiempo de servicio de la columna y la capacidad de remoción mejoran cuando la altura aumenta y el flujo volumétrico disminuye. El modelo del tiempo de servicio de la altura del lecho (BDST) se aplicó a los datos experimentales ajustándose de manera aceptable.

Palabras Clave: Carbón activado, Colorantes, Isotermas de adsorción, Curvas de ruptura.

\begin{abstract}
Dyes are usually present in water effluents of many industries, such as textiles, leather, paper, printing and cosmetics. The effectiveness of the adsorption of dyes in wastewater using inexpensive adsorbents has enabled the extension of this technique for removal, over other methods of treatment. This study investigated the adsorption capacity of a commercial activated carbon (AC) on the removal of reactive dye Blue 39 (B39) in water solution at $25^{\circ} \mathrm{C}$. The experimental, conform to the model of Freundlich isotherm, presenting a maximum adsorption capacity monolayer $17.7 \mathrm{mg} \mathrm{g}^{-1}$ at $\mathrm{pH}$. The dynamic study at different heights of the bed $(1,3$ and $5 \mathrm{~cm})$, flow rates $\left(1\right.$ and $5 \mathrm{~cm}^{3} \mathrm{~min}^{-1}$ ) and initial concentration of $5,5 \mathrm{mg} \mathrm{dm}^{-3}$, shows that the time service and the capacity column removal improved when the height increases and the flow rate decreases. The model of the service time of the bed height (BDST) was applied to the experimental data at an acceptable conform.
\end{abstract}

Keywords: Activated charcoal, Dyes, Adsorption isotherms, Curves break. 


\section{INTRODUCCIÓN}

Las industrias como la textil, cuero, papel, plásticos, y otras, usan tintes para sus productos, como resultado de esta actividad se genera una considerable cantidad de aguas residuales. Se estima que más de 100,000 colorantes están disponibles en el mercado, y más de 700,000 toneladas métricas de colorante se producen anualmente, de los cuales el 10 al 15\% se desechan en los efluentes provenientes de las operaciones de teñido [1, 2]. Estas descargas de aguas coloreadas a los cuerpos de agua causa serios problemas al medio ambiente, tales como, la disminución de la actividad fotosintética debido a la interferencia en la penetración de la luz [3], e inhiben la reacción de los agentes oxidantes [4]; es importante además señalar, que algunos colorantes tienen efecto tóxico, cancerígeno y mutagénico por la tendencia a formar quelatos de iones metálicos que producen microtoxocidad tanto para la vida acuática como humana [5].

La adsorción es una de las técnicas más empleadas en la remoción de colorantes, en virtud de la simplicidad del diseño, facilidad de operación e insensibilidad a las sustancias tóxicas [6]. Los carbones activados, por su gran porosidad, son empleados ampliamente como adsorbentes en las operaciones industriales de purificación y recuperación química, lo cual se debe a su extensa área superficial entre 500 y $2000 \mathrm{~m}^{2} \mathrm{~g}^{-1}$, su gran volumen de poro y la presencia de grupos funcionales superficiales, especialmente grupos oxigenados [7].

Los estudios de adsorción en disolución mediante experimentos por lotes (discontinuos) proporcionan una medida de la eficacia de la adsorción en la eliminación de sustancias específicas, así como la capacidad máxima de adsorción. Tales experimentos consisten en colocar una cantidad determinada de adsorbente en contacto con la disolución que contiene la sustancia que se va a remover, manteniéndola en suspensión mediante agitación el tiempo necesario para alcanzar el equilibrio. Los estudios de adsorción en una columna de lecho fijo, a diferencia de los experimentos por lotes, no funcionan bajo condiciones de equilibrio debido a que continuamente ingresa a la columna una disolución de alimentación, donde se establece un proceso de transferencia de masa permanente entre una fase móvil que contiene el adsorbible (sustancia a remover) y la fase sólida del lecho adsorbente. Los procesos industriales generalmente se trabajan bajo condiciones continuas, por lo tanto este tipo de estudios proporciona la aplicación más práctica en el tratamiento de aguas residuales [8].

En la figura 1 se muestra el esquema de una columna empacada con un material adsorbente en contacto con un líquido que contiene un adsorbible de concentración. Inicialmente la parte inferior del adsorbente remueve rápidamente el soluto, así que el fluido que sale de la columna está prácticamente libre de adsorbible. Como el volumen de líquido que atra- viesa la columna aumenta, la zona de transferencia de masa (MTZ) comienza a desplazarse y la concentración del adsorbible a la salida aumenta con el tiempo. Se denomina punto de ruptura () al valor máximo en la concentración del adsorbible que se puede permitir a la salida de la columna, y al tiempo empleado para alcanzar esta concentración específica se le llama tiempo de ruptura $\left(t_{\mathrm{b}}\right)$ para cada una de las condiciones de operación de la columna. La curva que resulta de graficar la relación en función del tiempo se conoce como curva de ruptura. En este estudio el criterio de "tiempo de ruptura" se adopta como el lapso que transcurre cuando la concentración del adsorbible llegue al 10\% de la concentración inicial. Cuando la zona de transferencia de masa sale de la columna, el lecho está completamente saturado y la concentración a la salida es la misma de la entrada $[8,9]$. Los datos experimentales de este estudio pueden ajustarse a modelos matemáticos que permiten predecir el tiempo de servicio de la columna. Uno de los modelos ampliamente aplicado al carbón activado es el BDST (Bed Depth Service Time):

Este modelo supone que la difusión intraparticular y la resistencia de masa externa son insignificantes y que la cinética de adsorción está controlada por la reacción superficial entre el soluto en la disolución y el adsorbente que no ha sido usado [10]. La ecuación inicial propuesta por Bohart y Adams está dada por la expresión:

$$
\ln \left(\frac{C_{o}}{C}-1\right)=\ln \left(\mathrm{e}^{\left(K N_{o} Z\right)}-1\right)-K C_{o} t
$$

Figura 1. Curva de ruptura típica del carbón activado mostrando el movimiento de la zona de transferencia de masa. Tomado y adaptado de la referencia [9].

Figure 1. Typical breakthrough curve of activated carbon showing movement of the mass transfer zone. Taken and adapted from reference [9].

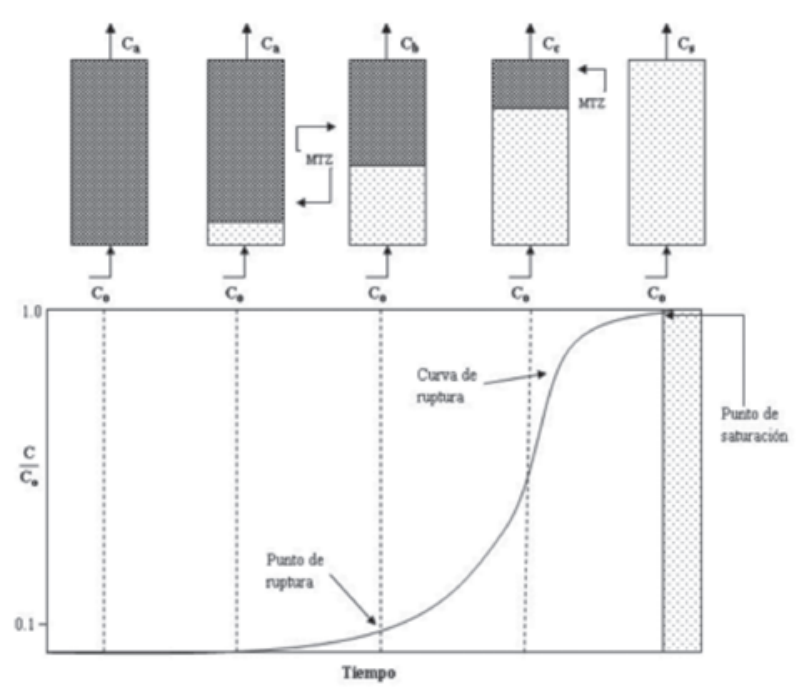


En esta relación $\mathrm{e}^{\left(K N_{\circ} \frac{Z}{U}\right)}>>1$, por lo tanto $\ln \left(\mathrm{e}^{\left(K N_{o} \frac{Z}{T}\right)}-1\right)$ $\cong \operatorname{Ine}^{\left(K N_{\circ} \frac{Z}{T}\right)}$ esta es la razón por la cual Hutchins [11] propuso la siguiente relación lineal entre la altura del lecho de la columna $(Z)$ y el tiempo de servicio : $(t)$

$$
t=\frac{N_{o}}{C_{o} U} Z-\frac{1}{K C_{o}} \ln \left(\frac{C_{o}}{C}-1\right)
$$

donde $t$ es el tiempo de servicio de la columna en el punto de ruptura $(\mathrm{min}), N_{o}$ es la capacidad dinámica del lecho $\left(\mathrm{mg} \mathrm{dm}{ }^{-3}\right), Z$ es la altura del lecho $(\mathrm{cm}), U$ es la velocidad de flujo lineal $\left(\mathrm{cmmin}^{-1}\right)$ definida como la razón entre el flujo volumétrico $Q_{v}\left(\mathrm{~cm}^{3} \mathrm{~min}^{-1}\right)$ y el área de la sección transversal de la columna $\left(\mathrm{cm}^{2}\right), C_{0}$ y $C$ son, respectivamente la concentración inicial y a un tiempo $t\left(\mathrm{mg} \mathrm{dm}^{-3}\right) \mathrm{y}, K$ es la constante de velocidad de adsorción $\left(\mathrm{dm}^{3} \mathrm{~min}^{-1} \mathrm{mg}^{-1}\right)$ [11].

La ecuación 2 se conoce con el nombre de tiempo de servicio de una altura de lecho (BDST) y representa una línea recta que puede expresarse de una manera simplificada como:

donde $\quad t=a Z+b$

$$
t=a Z+b
$$

$$
\mathrm{y} \quad b=\frac{1}{K C_{o}} \ln \left(\frac{C_{o}}{C}-1\right)
$$

La capacidad dinámica del lecho, puede evaluarse a partir de la pendiente de la gráfica y la constante de velocidad de adsorción, puede calcularse a partir de la ordenada al origen.

Esta investigación evaluó la capacidad de adsorción de un carbón activado comercial marca Norit ${ }^{\circledR}$ en la remoción del colorante B39 en disolución acuosa, modificando el pH y la concentración inicial en un estudio por lote; de igual manera, también se evaluó el efecto de varias condiciones experimentales como: el flujo volumétrico () y la altura del lecho (), sobre la capacidad de adsorción en columna en un estudio dinámico, manteniendo la temperatura y $\mathrm{pH}$ constantes. Los resultados obtenidos se ajustaron a los modelos de Langmuir, Freundlich y el de predicción del tiempo de servicio de la columna BDST.

\section{METODOLOGÍA}

\subsection{Adsorbato}

El colorante Blue 39 (B39) empleado en este estudio fue suministrado por la empresa Minerva Color Colombiana
Ltda. (Bogotá, Colombia). Su fórmula molecular es $\mathrm{C}_{25} \mathrm{H}-$ ${ }_{14} \mathrm{ClN}_{10} \mathrm{Na}_{5} \mathrm{O}_{16} \mathrm{~S}_{5}$ y presenta una longitud de onda máxima en $610 \mathrm{~nm}$. La disolución "stock" de colorante (1000 mg $\mathrm{dm}^{-3}$ ) se preparó disolviendo la cantidad requerida de B39 en agua destilada. Las disoluciones utilizadas se prepararon por dilución a parir de la "stock". La estructura molecular del B39 se muestra en la figura 1.

Figura 1. Estructura molecular del colorante B39. Figure 1. Molecular structure of dye B39.

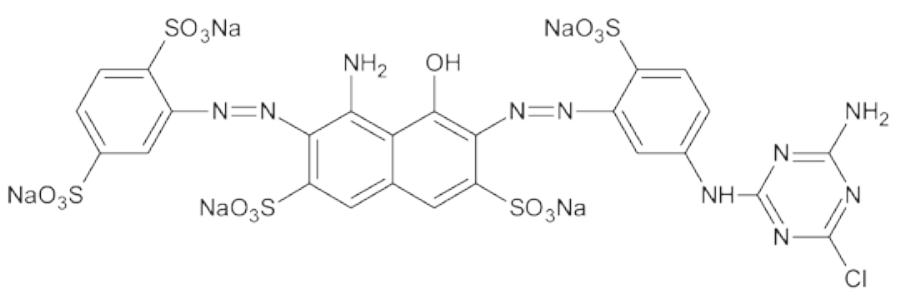

\subsection{Adsorbente}

Se seleccionó un carbón activado granular (GAC) comercial marca Norit ${ }^{\circledR}$, empleado industrialmente en el tratamiento de aguas residuales para la remoción de sustancias orgánicas y metales pesados. Previo a su uso el adsorbente se lavó con agua destilada y posteriormente se secó a $100^{\circ} \mathrm{C}$ por 12 horas [8]. Luego se utilizó sin tratamientos posteriores.

\subsection{Caracterización del carbón activado}

El área superficial de BET (Brunauer-Emmett-Teller) se evaluó a partir de la isoterma de adsorción de $\mathrm{N}_{2}$ a $77 \mathrm{~K}$ empleando un sortómetro Micromeritics Gemini VII 2390. El contenido de humedad, materia volátil, cenizas y carbono fijo, se determinaron de acuerdo con la metodología establecida en la norma ASTM D 3173-75. El contenido de carbono, nitrógeno, hidrógeno y oxígeno (por diferencia) se determinaron de acuerdo con la metodología establecida en la norma ASTM 5373, y azufre total por la norma ASTM D 4239 (método B). Estos análisis fueron realizados en laboratorios externos a la Universidad.

\subsection{Estudio por lote}

A partir de la disolución "stock" se realizaron diluciones de concentraciones entre los $5 \mathrm{mg} \mathrm{dm}^{-3}$ hasta los 200 $\mathrm{mg} \mathrm{dm}{ }^{-3}$, ajustándole el $\mathrm{pH}$ a valores comprendidos entre $6,0-8,0 \pm 0,2$ con $\mathrm{HCl}$ y $\mathrm{NaOH} 0,1 \mathrm{M}$ según conveniencia. A $1000 \mathrm{mg}$ del carbón activado se le adicionaron $100 \mathrm{~cm}^{3}$ de cada una de las diluciones, colocándolas luego en un agitador horizontal a $120 \mathrm{rpm}$ por 12 horas, a la temperatura del laboratorio $25^{\circ} \mathrm{C} \pm 1^{\circ} \mathrm{C}$. Finalizada la agitación, 
se separó el carbón activado por filtración y se analizó la concentración de B39 en un espectrofotómetro marca THERMO ELECTRON modelo GENESYS 2, empleando una curva de calibración.

\subsection{Estudio dinámico en columna}

El arreglo experimental del estudio dinámico consistió en un depósito de almacenamiento que contiene la disolución de B39, está a su vez se alimenta a una columna de vidrio de $2,0 \mathrm{~cm}$ de diámetro interno, empacada con carbón activado granular. Para un caudal determinado $\left(1,0\right.$, y $\left.5,0 \mathrm{~cm}^{3} \mathrm{~min}^{-1}\right)$, a dos alturas diferentes $(1$ y $5 \mathrm{~cm})$ y a la concentración inicial de $5,5 \mathrm{mg} \mathrm{dm}^{-3}$, en intervalos regulares de tiempo, usando una jeringa con una aguja hipodérmica, se tomaron alícuotas del fluido a la salida del lecho de carbón activado, con el propósito de determinar el contenido del colorante B39, para tal efecto, se empleó un espectrofotómetro marca THERMO ELECTRON modelo GENESYS 2. La disolución después de pasar por la columna se descargó en un tanque colector. Los flujos volumétricos se regularon con la ayuda de una bomba peristáltica.

Tanto para los experimentos por lote y dinámico se planteó un diseño factorial tomando las variables correspondientes, realizando los ensayos por triplicado.

\section{RESULTADOS Y DISCUSIÓN}

\subsection{Caracterización del carbón activado}

Los resultados del análisis próximo, análisis elemental y de textura se muestran en la tabla 1.

Tabla 1. Principales características del carbón activado

Table 1. Main characteristics of the activated coal

\begin{tabular}{|l|l|}
\hline \multicolumn{1}{|c|}{ Parámetro } & \multicolumn{1}{c|}{ Valor } \\
\hline Área superficial $\left(\mathrm{m}^{2} \mathrm{~g}^{-1}\right)($ basado en BET) & 586 \\
\hline Volumen total de poro $\left(\mathrm{cm}^{3} \mathrm{~g}^{-1}\right)$ & 0,67 \\
\hline Tamaño de poro $(\AA)$ & 46,0 \\
\hline Contenido de cenizas (base seca) $(\%)$ & 14,5 \\
\hline Contenido de humedad (\%) & 5,9 \\
\hline Materia volátil $(\%)$ & 8,2 \\
\hline Análisis elemental $(\%)$ & \\
\hline $\mathrm{C}$ & 77,2 \\
\hline $\mathrm{H}$ & 2,8 \\
\hline $\mathrm{N}$ & 0,9 \\
\hline $\mathrm{O}$ (por diferencia) & 3,9 \\
\hline $\mathrm{S}$ & 0,7 \\
\hline
\end{tabular}

Los resultados de textura muestran un carbón activado con una baja área superficial y aunque se conoce que la capacidad de adsorción depende además del área superficial, de la presencia de microporosidad y de los fenómenos de oclusión entre otros, también depende y no menos importante, de la química de superficie. La presencia de heteroátomos principalmente oxígeno (tabla 1), que forma grupos funcionales orgánicos tales como ácidos carboxílicos (también en la forma de su anhídrido cíclico), lactonas, lactoles y grupos hidroxílicos de carácter fenólico, son los responsables de las diferentes interacciones que se establecen entre el colorante y el carbón activado.

De otra parte, no existe mucha evidencia sobre el efecto, en la capacidad de adsorción, del contenido de cenizas del carbón; en perspectiva, los efectos de estos componentes dependen de la carga, de la especiación y de las características de textura de los óxidos que configuran las cenizas del carbón. La posible acción de la humedad es un aspecto más difícil de evaluar como quiera que el adsorbente se carga a la columna bajo condiciones anhidras [8].

\subsection{Isotermas de adsorción}

\subsubsection{Isoterma de Langmuir}

La isoterma de adsorción de Langmuir es una de las más empleadas en los estudios de adsorción por lote, según reportes de científicos se usa ampliamente en el ajuste de datos experimentales relacionados con la eliminación de metales pesados, colorantes y contaminantes orgánicos por adsorción sobre carbón activado, arcilla, residuos agrícolas, entre otros [12]. Este modelo asume que la adsorción ocurre en sitios específicos de la superficie del adsorbente y en cada uno de ellos se puede acomodar sólo una molécula, para una cobertura en monocapa. Además, las moléculas adsorbidas no pueden migrar a través de la superficie o interactuar con las moléculas vecinas y la energía de adsorción es la misma en todos los sitios [12,13]. A partir del modelo de isoterma de Langmuir se puede estimar la capacidad máxima de adsorción producida por el cubrimiento completo en monocapa de la superficie del adsorbente. La forma lineal del modelo se expresa como sigue [14]:

$$
\frac{C_{e}}{q}=\frac{1}{b q_{\operatorname{mac}}}+\frac{C_{e}}{q_{\max }}
$$

donde $q$ es la cantidad de soluto removido por unidad de masa de adsorbente $\left(\mathrm{mg} \mathrm{g}^{-1}\right), C_{e}$ es la concentración del adsorbible en el equilibrio $\left(\mathrm{mg} \mathrm{dm}^{-3}\right), \mathrm{y} q_{\max }$ son las constantes de Langmuir, y representan la capacidad máxima de adsorción del adsorbente $\left(\mathrm{mg} \mathrm{g}^{-1}\right)$ y la cons- 
tante de energía relacionada con el calor de adsorción respectivamente $\left(\mathrm{dm}^{3} \mathrm{mg}^{-1}\right)$. Estos valores se pueden obtener graficando $\mathrm{Ce}$ versus $\mathrm{Ce} / \mathrm{q}$ y aplicando regresión lineal, como se muestra en la figura 2 para los datos específicos del estudio.
En la tabla 2 donde se resume la información obtenida. Se observa que $q_{\max }$ está en el rango entre 16,5 y $17,7 \mathrm{mg} \mathrm{g}^{-1}$ y $b$ alrededor de $0,5 \mathrm{dm}^{3} \mathrm{mg}^{-1}$ para todos los $\mathrm{pH}$ estudiados. Estos valores muestran que el carbón activado empleado puede considerarse como una alternativa para la remoción de colorantes.

Tabla 2. Constantes de los modelos de Langmuir y Freundlich a diferentes pH.

Table 2. Constants Langmuir and Freundlich models at different pH.

\begin{tabular}{|c|c|c|c|c|c|c|}
\hline & $\begin{array}{c}\text { Constante de } \\
\text { Langmuir }\end{array}$ & $\begin{array}{c}\text { Constante de } \\
\text { Freundlich }\end{array}$ & & \\
\hline $\mathbf{p H}$ & $\begin{array}{c}\text { Coeficiente de } \\
\text { correlación } \\
\left(\boldsymbol{R}^{2}\right)\end{array}$ & $\left(\mathrm{mg} \mathrm{g}^{-1}\right)$ & $\left(\mathrm{dm} \mathrm{mg}^{-1}\right)$ & $\begin{array}{c}\text { Coeficiente de } \\
\text { correlación } \\
\left(\boldsymbol{R}^{2}\right)\end{array}$ & & \\
\hline $\mathbf{6}$ & 0,96 & 16,5 & 0,5 & 1,00 & 3.7 & 2,4 \\
\hline $\mathbf{7}$ & 0,95 & 17,0 & 0,4 & 0,95 & 3,1 & 2,1 \\
\hline $\mathbf{8}$ & 0,96 & 17,7 & 0,5 & 0,98 & 3,4 & 2,1 \\
\hline
\end{tabular}

Figura 2. Comparación entre la ecuación linealizada del modelo de Langmuir y los datos experimentales a diferentes pH. a) $\mathrm{pH}=6, \mathrm{~b}) \mathrm{pH}=7$ y c) $\mathrm{pH}=8$.

Figure 2. Comparison between the linear equation Langmuir model and the experimental data at different $\mathrm{pH}$. a) $\mathrm{pH}=$ $6, \mathrm{~b}) \mathrm{pH}=7$ and c) $\mathrm{pH}=8$.

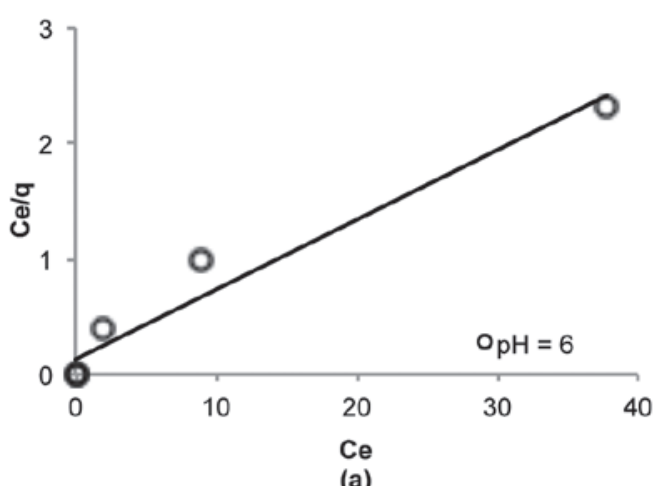

(a)

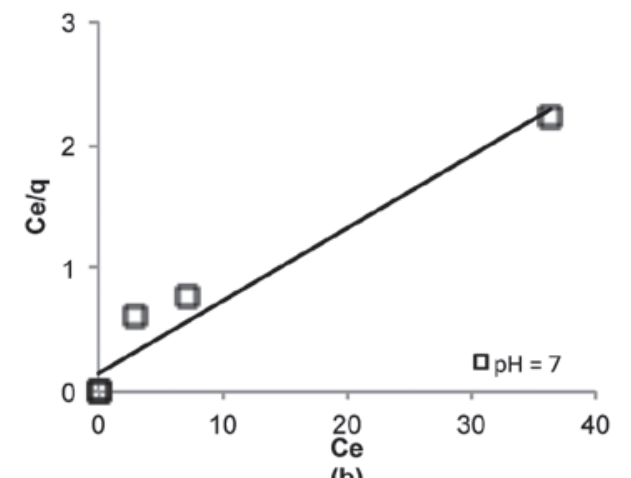

(b)

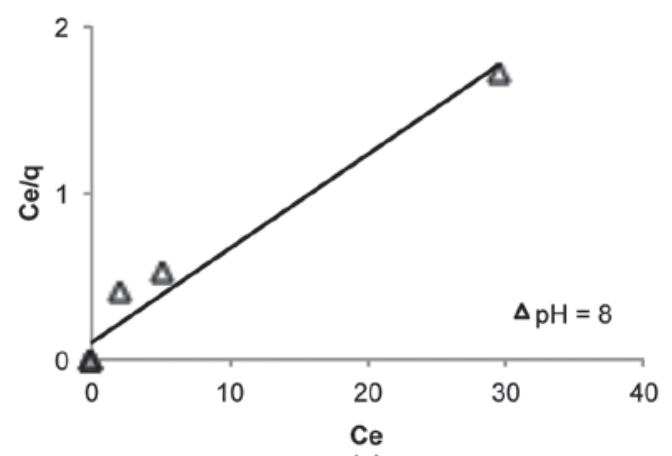

(c) 


\subsubsection{Isoterma de Freundlich}

Este modelo supone que la superficie del adsorbente es heterogénea y que los sitios de adsorción tienen distintas afinidades; en primer lugar, se ocupan las posiciones de mayor afinidad y posteriormente se va ocupando el resto, para un crecimiento en multicapa. La forma linealizada del modelo de Freundlich se expresa de la siguiente manera [15]:

$$
\ln q=\ln k+\frac{1}{n} \ln C_{e}
$$

donde $q$ es la cantidad de soluto removido por unidad de masa de adsorbente $\left(\mathrm{mg} \mathrm{g}^{-1}\right), \mathrm{Ce}$ es la concentración del adsorbible en el equilibrio $\left(\mathrm{mg} \mathrm{dm}^{-3}\right), k$ es la constante de equilibrio $\left(\mathrm{mg} \mathrm{g}^{-1}\left(\mathrm{dm}^{3} \mathrm{mg}^{-1}\right)^{-1 / 1}\right)$ y $n$ es una constante relacionada con la afinidad entre el adsorbente y el soluto. Representando gráficamente $\operatorname{Inq}$ versus $\operatorname{InC} C_{e}$ y aplicando regresión lineal, de los valores de la pendiente y la ordenada al origen, se obtienen los parámetros de la isoterma de Freundlich. La figura 3 muestra los resultados del ajuste de los datos experimentales al modelo y en la tabla 2 aparecen los valores de los parámetros.
Para determinar el modelo de isoterma que mejor se ajustara a los datos de equilibrio experimentales, se comparó los coeficientes de correlación de ambos modelos. Los valores de $R^{2}$ para las isotermas de Langmuir y Freundlich fueron 0,96 y 1,0 respectivamente a $\mathrm{pH} 6$, similar comportamiento se muestra para los otros $\mathrm{pH}$ estudiados. A pesar de que los valores están muy cercanos, se sugiere que la isoterma de Freundlich se ajusta mejor al proceso de adsorción que la isoterma de Langmuir. Puede asumirse entonces, que en el CA predominan los sitios activos que tienen una energía de adsorción diferente que estimulan la formación de multicapas de moléculas de B39, posiblemente de naturaleza física como lo indica el valor de por encima de la unidad [12]. Pero el ajuste satisfactorio al modelo de Langmuir sugiere que también existen sitios activos que presentan una distribución homogénea de la energía de adsorción, que tienden a formar monocapas de moléculas de B39 sobre el CA.

\subsubsection{Efecto del $\mathrm{pH}$}

El efecto del pH inicial de la disolución sobre el porcentaje de remoción a una concentración inicial de $200 \mathrm{mg} \mathrm{dm}^{-3} \mathrm{y}$ dosis de adsorbente de $10 \mathrm{~g} \mathrm{dm}^{-3}$, se muestra en la figura 4. Se observa que en el rango de $\mathrm{pH}$ estudiado (6-8) los

Figura 3. Comparación entre la ecuación linealizada del modelo de Freundlich y los datos experimentales a diferentes $\mathrm{pH}$. a) $\mathrm{pH}=6, \mathrm{~b}) \mathrm{pH}=7$ y c) $\mathrm{pH}=8$.

Figure 3. Comparison between the linear equation Freundlich model and the experimental data at different $\mathrm{pH}$. a) $\mathrm{pH}$ $=6, \mathrm{~b}) \mathrm{pH}=7$ and c) $\mathrm{pH}=8$.
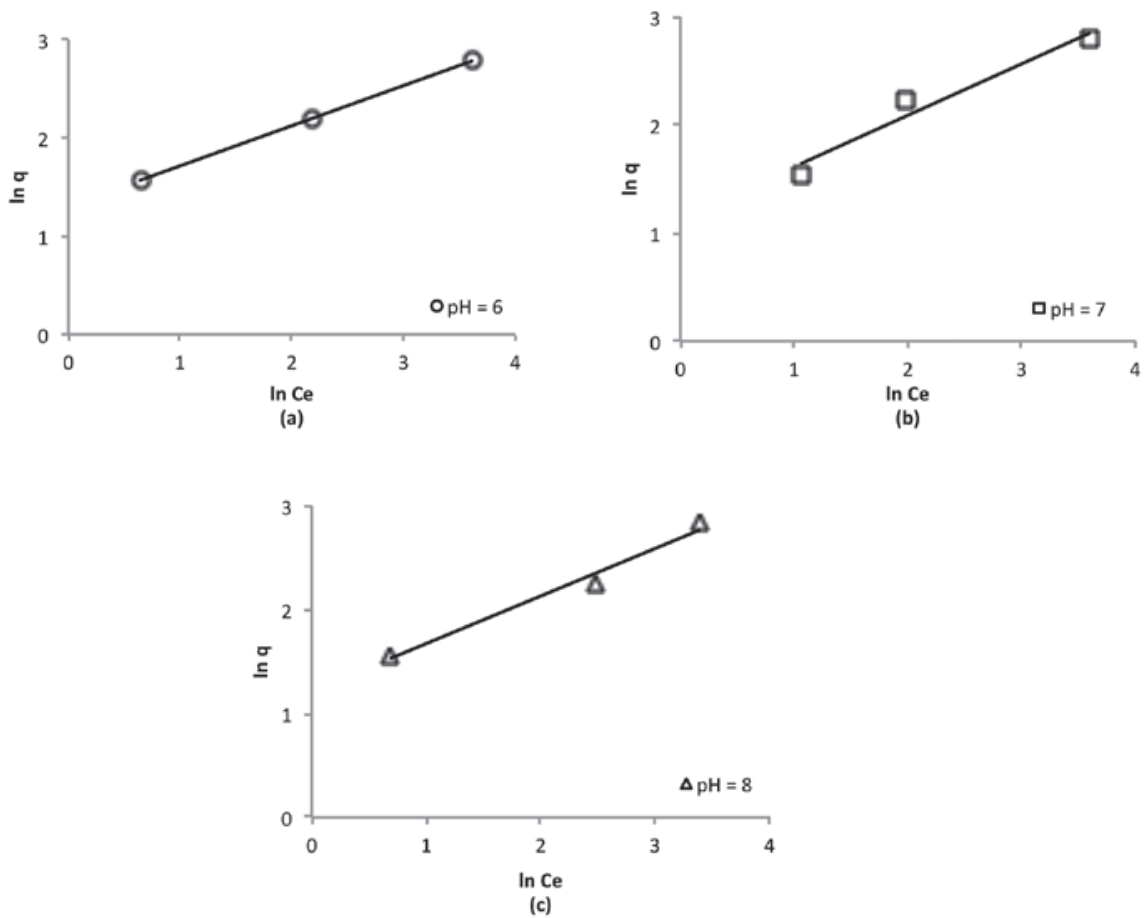
cambios no son tan significativos en el porcentaje de remoción del colorante B 39; un comportamiento similar se registra para otras concentraciones iniciales.

Figura 4. Influencia del $\mathrm{pH}$ sobre el porcentaje de remoción a una $200 \mathrm{mg} \mathrm{dm}^{-3}$.

Figure 4. Influence of $\mathrm{pH}$ on the rate removal to $\mathrm{a}=200$ $\mathrm{mg} \mathrm{dm}{ }^{-3}$.

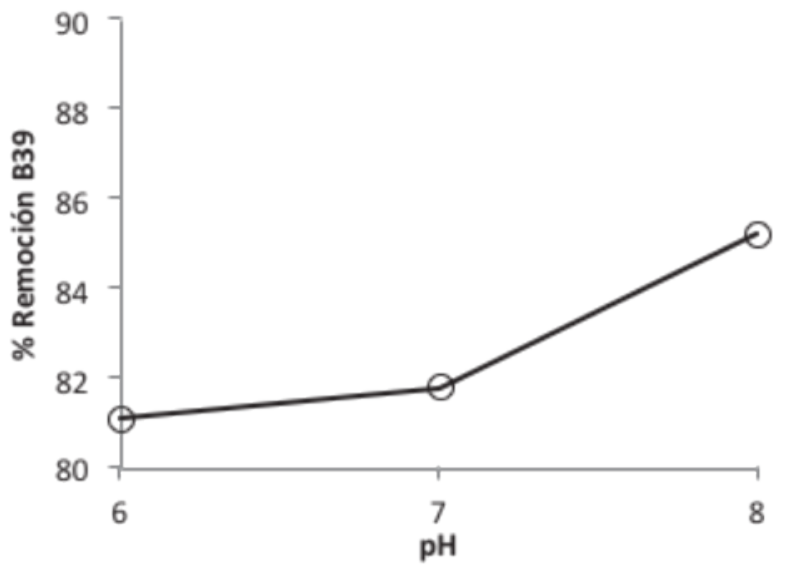

Es posible que en la región ácida la presencia de iones $\mathrm{H}^{+}$ en la disolución compita con las moléculas del colorante B 39 por los sitios de unión del CA y como resultado, se reduce el valor de la capacidad de adsorción. La presencia de iones hidroxilo en la región básica promueve las atracciones electrostáticas, mejorando la capacidad adsorbente. Pero independiente del $\mathrm{pH}$ de la disolución, la adsorción de los colorantes sobre carbón activado se favorecen, en primer lugar, a las fuerzas de dispersión y a las interacciones entre los electrones $\pi$ de los sitios ácidos de Lewis en el plano basal del carbón activado y los electrones libres de las moléculas de B39 presentes en los anillos aromáticos y enlaces múltiples, y en segundo lugar, a los enlaces de hidrógeno [16]. Sin embargo, existen esfuerzos y expecta- tivas por parte del grupo de trabajo evaluar el potencial de carga cero y su incidencia en el proceso.

\subsection{Estudio dinámico en columna}

\subsubsection{Capacidad de adsorción en columna}

La cantidad de colorante adsorbido por gramo de carbón activado en el punto de ruptura, $q_{b}\left(\mathrm{mg} \mathrm{g}^{-1}\right)$, se obtuvo a partir de la siguiente ecuación:

$$
q_{b}=\frac{Q_{v} t_{10 \%} C_{o}}{1000 m}
$$

donde $t_{10 \%}$ es el tiempo de servicio (min) obtenido cuando la concentración del colorante a la salida de la columna es el $10 \%$ de la concentración inicial o de entrada, $C_{0}$ es la concentración inicial o de entrada a la columna (mg dm$\left.{ }^{3}\right), Q_{v}$ es el flujo volumétrico $\left(\mathrm{cm}^{3} \mathrm{~min}^{-1}\right), m$ es la masa de adsorbente y el valor de 1000 corresponde a un factor de conversión [9]. La tabla 3 muestra los resultados a diferentes condiciones de operación de la columna. Se observa que la máxima capacidad de adsorción alcanzada fue de $0,6 \mathrm{mg} \mathrm{g}^{-1}$ a una altura del lecho de $5 \mathrm{~cm}$, un flujo volumétrico de $1 \mathrm{cmmin}^{-1}$ y una concentración inicial de $5,5 \mathrm{mg}$ $\mathrm{dm}^{-3}$. Este resultado muestra una reducción significativa, comparado con el valor que reporta el modelo de Langmuir $\left(17,7 \mathrm{mg} \mathrm{g}^{-1}\right)$.

La diferencia significativa entre los valores de la capacidad de adsorción del adsorbente en los dos estudios realizados se debe a que en un proceso por lote el carbón activado permanece más tiempo en contacto con la disolución, esta situación permite que se alcance una condición de equilibrio; pero además, la agitación permanente proporciona una mejor interacción entre los sitios activos del adsorbente y el colorante, lo cual favorece la velocidad de transferencia de masa y, por lo tanto, la mayor retención.

Tabla 3. Resultados de la capacidad de adsorción en columna.

Table 3. Results of column adsorption capacity.

\begin{tabular}{|c|c|c|c|c|c|}
\hline $\begin{array}{c}\text { Concentración inicial } \\
\left(\mathrm{mg} \mathrm{dm}^{-3}\right)\end{array}$ & $\begin{array}{l}\text { Tiempo de rup- } \\
\text { tura (min) }\end{array}$ & $\begin{array}{l}\text { Flujo Volumétrico } \\
\left(\mathrm{cmmin}^{-1}\right)\end{array}$ & $\begin{array}{l}\text { Altura del lecho } \\
(\mathrm{cm})\end{array}$ & $\begin{array}{l}\text { Masa de lecho } \\
\text { (g) }\end{array}$ & $\begin{array}{l}\text { Capacidad de adsorción } \\
\left.\text { de la columna ( } \mathrm{mg} \mathrm{g}^{-1}\right)\end{array}$ \\
\hline 5,5 & 408 & 1 & 5 & 3,5 & $6 \times 10^{-1}$ \\
\hline 5,5 & 104 & 1 & 3 & 2,1 & $3 \times 10^{-1}$ \\
\hline 5,5 & 4 & 1 & 1 & 0,7 & $3 \times 10^{-2}$ \\
\hline 5,5 & 81 & 5 & 5 & 3,5 & $1 \times 10^{-1}$ \\
\hline 5,5 & 21 & 5 & 3 & 2,1 & $5 \times 10^{-2}$ \\
\hline 5,5 & 0,01 & 5 & 1 & 0,7 & $4 \times 10^{-4}$ \\
\hline
\end{tabular}




\subsubsection{Efecto de la altura del lecho}

La figura 5 muestra las curvas de ruptura obtenidas para diferentes alturas del lecho 1,3 y $5 \mathrm{~cm}$, a un flujo volumétrico constante de $1 \mathrm{~cm}^{3} \mathrm{~min}^{-1}$ y una concentración de entrada de $5,5 \mathrm{mg} \mathrm{dm}^{-3}$. Bajo estas condiciones se encontró que el tiempo de ruptura aumenta de 4 a 408 minutos cuando se incrementa la altura de 1 a $5 \mathrm{~cm}$. Un comportamiento similar se registra para otras condiciones de operación estudiadas. Esto indica, que al aumentar la altura del lecho mayor es la eficiencia de eliminación del colorante B 39 en la columna, disminuyendo la concentración en el efluente.

Algo importante que se observa de las pendientes de las curvas de ruptura, es que disminuyen con el aumento de la altura, lo que resulta en una zona de transferencia de masa más amplia [17]. Pero además, un aumento en la altura del lecho trae como consecuencia un incremento en el área superficial del adsorbente, disponiéndose por lo tanto, de más sitios de unión para la adsorción [9].

Figura 5. Curvas de ruptura para la adsorción de colorante B39 a diferentes alturas de la columna y flujo volumétrico de $1 \mathrm{cmmin}^{-1}$.

Figure 5. Breakthrough curves for adsorption of dye B39 at different heights of the column and flow rate of $1 \mathrm{~cm}^{3}$ $\min ^{-1}$.

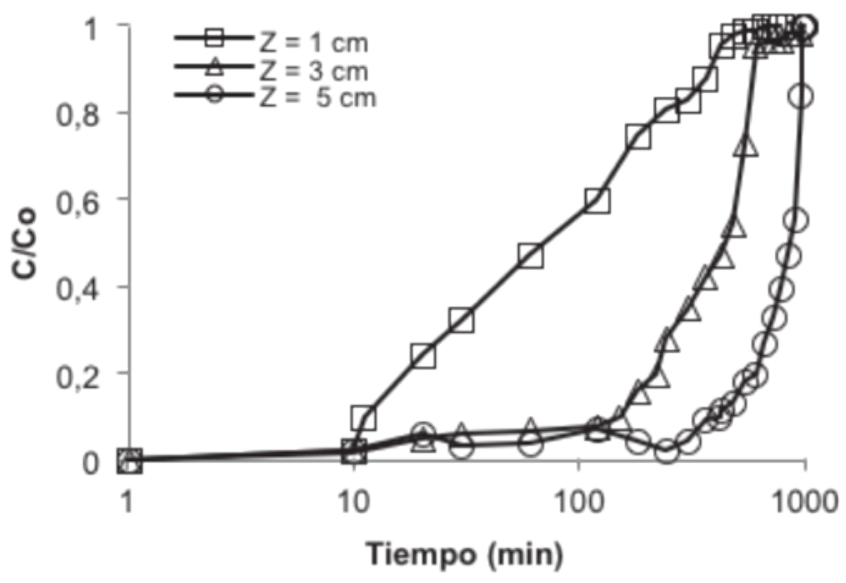

\subsubsection{Efecto del flujo volumétrico}

Otro de los parámetros que afecta la remoción de colorantes en una columna de lecho fijo, es el flujo volu- métrico. Por ello, se realizaron experimentos con dos flujos, 1 y $5 \mathrm{~cm}^{3} \mathrm{~min}^{-1}$ y tres alturas del lecho. Para una concentración inicial de $5,5 \mathrm{mg} \mathrm{dm}^{-3}$ y una altura del lecho de $5 \mathrm{~cm}$ el tiempo de ruptura cambia de 81 a 408 minutos cuando el flujo volumétrico varía de 5 a $1 \mathrm{~cm}$ ${ }^{3} \mathrm{~min}^{-1}$ (figura 6). Una tendencia similar se observa para las otras condiciones de operación de la columna. Este resultado indica que el tiempo de ruptura es significativamente mayor con una disminución en el flujo volumétrico, este comportamiento ha sido reportado por otros autores [9, 17,18].

La razón de esta variación es que a mayor flujo volumétrico la tasa de transferencia de masa se incrementa, es decir, la velocidad con que el colorante es adsorbido por unidad de altura del lecho (zona de transferencia de masa), en consecuencia el carbón activado se satura más rápido.

Figura 6. Curvas de ruptura para la adsorción de colorante B39 en una columna empacada a diferentes flujos volumétricos y un lecho de $5 \mathrm{~cm}$ de altura.

Figure 6. Breakthrough curves for adsorption of dye B39 in a packed column at different flow rates and a bed height of $5 \mathrm{~cm}$.

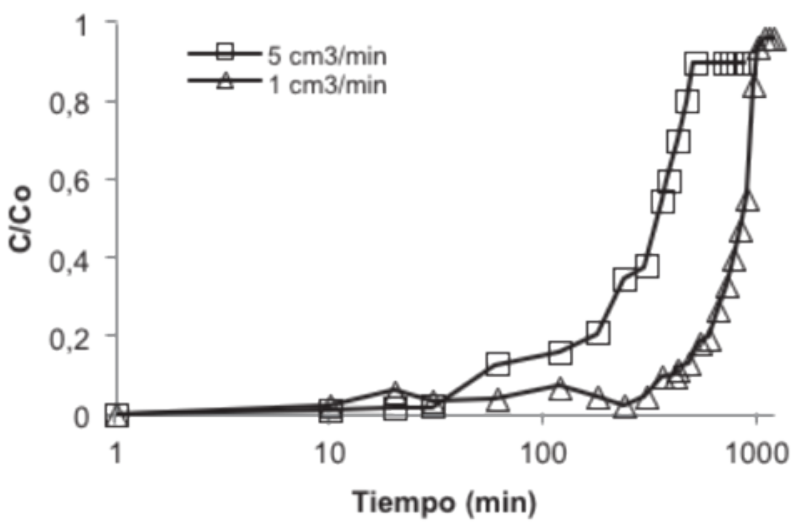

\subsubsection{Aplicación del modelo BDST}

Los parámetros del modelo BDST y se obtuvieron graficando $Z$ contra $t$ para diferentes relaciones de $(0,1,0,2$, $0,4$, y 0,6$)$, velocidades de flujo lineal de 0,31 y $1,6 \mathrm{~cm} \mathrm{~min}^{-1}$ (flujo volumétrico de 1,0 y $5,0 \mathrm{~cm}^{3} \mathrm{~min}^{-1}$ respectivamente) y concentración inicial de $5,5 \mathrm{mg} \mathrm{dm}^{-3}$. Los coeficientes de correlación obtenidos $\left(R^{2}>0,94\right)$ son satisfactorios e indican la validez del modelo BDST para el sistema en estudio. En La tabla 4 se muestran los resultados. 
Tabla 4. Parámetros del modelo BDST.

Table 4. BDST model parameters.

\begin{tabular}{|c|c|c|c|c|c|}
\hline$(\mathrm{mg} \mathrm{dm}-3)$ & & $\left(\mathrm{cm} \mathrm{min} \mathrm{min}^{-1}\right)$ & $(\mathrm{mg} \mathrm{dm}-3)$ & $\left(\mathrm{dmmin}^{-1} \mathrm{mg}^{-1}\right)$ & $\mathbf{R}$ \\
\hline \multirow[t]{5}{*}{5,5} & 0,1 & 0,31 & 179 & $3,5 \times 10^{-3}$ & 0,97 \\
\hline & 0,2 & 0,31 & 246 & $1,6 \times 10^{-3}$ & 0,97 \\
\hline & 0,4 & 0,31 & 315 & $4,2 \times 10^{-4}$ & 0,98 \\
\hline & 0,6 & 0,31 & 327 & $1,0 \times 10^{-3}$ & 1,00 \\
\hline & 0,8 & 0,31 & 318 & $1,2 \times 10^{-2}$ & 0,99 \\
\hline \multirow[t]{5}{*}{5,5} & 0,1 & 1,6 & 178 & $1,5 \times 10^{-2}$ & 0,94 \\
\hline & 0,2 & 1,6 & 350 & $5,0 \times 10^{-3}$ & 0,95 \\
\hline & 0,4 & 1,6 & 627 & $8,6 \times 10^{-4}$ & 0,97 \\
\hline & 0,6 & 1,6 & 785 & $1,0 \times 10^{-3}$ & 0,98 \\
\hline & 0,8 & 1,6 & 894 & $6,3 \times 10^{-3}$ & 1,00 \\
\hline
\end{tabular}

La capacidad de adsorción, es importante para la evaluación de la eficiencia de la adsorción, los valores obtenidos de este parámetro muestran que el carbón activado empleado es un adsorbente eficaz [19]. La constante de velocidad, , disminuye cuando se incrementa la velocidad lineal de flujo cuando se comparan las mismas relaciones de concentración $C / C_{o^{\prime}}$ esto muestra que la etapa inicial del proceso de adsorción se rige por la transferencia de masa externa [20].

\section{CONCLUSIONES}

- La capacidad de adsorción del carbón activado comercial empleado en la remoción del colorante B39 en disolución acuosa bajo condición discontinua mejora cuando aumenta el $\mathrm{pH}$.

- Los datos experimentales de equilibrio se ajustan significativamente al modelo de isoterma de Freundlich.

- La máxima capacidad de adsorción en condición discontinua fue de $17,7 \mathrm{mg} \mathrm{dm}^{-3}$ a $\mathrm{pH} 8$, resultado que es comparable con los valores reportados en la literatura científica.

- La máxima capacidad de adsorción en condición continua fue de $0,60 \mathrm{mg} \mathrm{g}^{-1}$ a una altura del lecho de $5 \mathrm{~cm}$, un flujo volumétrico de $1 \mathrm{~cm}^{3} \mathrm{~min}^{-1} \mathrm{y}$ una concentración de alimentación de $5,5 \mathrm{mg} \mathrm{dm}^{-3}$.

- Las curvas de ruptura obtenidas a diferentes condiciones de operación de la columna muestran que el tiempo de ruptura aumenta cuando se incrementa la altura del lecho y se disminuye el flujo volumétrico.

- El modelo BDST se ajustó de manera aceptable para las diferentes condiciones de altura y flujo volumétrico aplicado.

\section{Agradecimientos}

Los autores de este artículo agradecen la colaboración del Laboratorio de Química de la Universidad Autónoma del Caribe y el Laboratorio de Análisis Instrumental de la Universidad del Atlántico.

\section{REFERENCIAS}

[1] Tunc, Ö., Tanac, H., Aksu, Z., Potential use of cotton plant wastes for the removal of Remazol Black $B$ reactive dye, J. Hazard. Mater., 163,187-198, 2009.

[2] Dafale, N., Wate, S., Meshram, S., Nandy, T., Kinetic study approach of remazol black-B use for the development of two-stage anoxic-oxic reactor for decolorization/ biodegradation of azo dyes by activated bacterial consortium, J. Hazard. Mater., 159, 319-328, 2008.

[3] Choy, K. K. H., Porter, J. F., Mckay, G., Intraparticle diffusion in single and multicomponent acid dye adsorption from wastewater onto carbon. Chemical Engineering Journal., 103, 133-145, 2004.

[4] Ramakrishna, K., Viraraghavan, T., Use of slag for dye removal, Waste Management, 17, 483-488, 1997.

[5]Wang, S., Boyjoo, Y., Choueib, A., A comparative study of dye removal using fly ash treated by different methods, Chemosphere, 60, 1401-1407, 2005.

[6] Merzouk, B., Madani, K., Sekki, A., Treatment characteristics of textile wastewater and removal of heavy metals using the electroflotation technique, Desalination Journal, 228 (1-3), 245-254, 2008. 
[7] Bansal, R., C., Donnet, J. B., Stoeckli, F., Activated Carbon, Ed. Marcel Dekker (New York), 482p, 1988.

[8] Valencia, J. S., Castellar, G. C., Predicción de las curvas de ruptura para la remoción de plomo (II) en disolución acuosa sobre carbón activado en una columna empacada, Rev. Fac. Ing. Univ. Antioquia, 66, 141-158, 2013.

[9] Taty-Costodes, V. C., Fauduet, H., Porte, C., Ho. Y., Removal of lead (II) ions from synthetic and real effluents using immobilized Pinus sylvestris sawdust: Adsorption on a fixed-bed column, J. Hazard. Mater., B123, 135-144, 2005.

[10] Bohart, G. S., Adams, E. Q., Some aspects of the behavior of charcoal with respect to chlorine, J. Am. Chem. Soc., 42, 523-544, 1920.

[11] Hutchins, R. A., New method simplifies design of activated carbon systems, Am. J. Chem. Eng., 80, 133-138, 1973.

[12] Uma, Banerjee, S., Sharma, Y., Equilibrium and kinetic studies for removal of malachite green from aqueous solution by a low cost activated carbon, Journal of Industrial and Engineering Chemistry, 19, 1099-1105, 2013.

[13] Castellar, G. C., García, A. A., Remoción de $\mathrm{Pb}^{2+}$ en disolución acuosa sobre carbón activado en polvo: Estudio por lote, Prospect, 9, 59-68, 2011.
[14] Langmuir, I., Journal of the American Chemical Society, 40, 1361, 1918.

[15] Freundlich, H., Journal of Physical Chemistry, 57, 387, 1906.

[16] Órfão, J. J. M., Silva, A. I. M., Pereira, J. C. V., Barata, S. A., Fonseca, I. M., Faria, P. C. C., Pereira, M. F. R., Adsorption of a reactive dye on chemically modified activated carbons-influence of $\mathrm{pH}$. Colloid and Interface Science, 296, 480-489, 2006.

[17] Ahmad, A.A., Hameed, B.H., Fixed-bed adsorption of reactive azo dye onto granular activated carbon prepared from waste, J. Hazard. Mater., 175, 298-303, 2010.

[18] Ko, D.C.K., Porter, J.F., McKay, G. Optimized correlations for the fixed-bed adsorption of metal ions on bone char, Chem. Eng. Sci. 55, 5819-5829, 2000.

[19] Wenhong, L., Qinyan, Y., Peng, T., Zuohao,M., Baoyu, G., Jinze, L.,Xing, X., Adsorption characteristics of dyes in columns of activated carbon prepared from paper mill sewage sludge, Chemical Engineering Journal, 178, 197-203, 2011.

[20] Foo, K.Y., Hameed, B.H., Dynamic adsorption behavior of methylene blue onto oil palm shell granular activated carbon prepared by microwave heating. Chemical Engineering Journal, 203, 81-87, 2012. 